\title{
5. MAGNETIC, BATHYMETRIC, SEISMIC REFLECTION, AND POSITIONING DATA COLLECTED UNDERWAY ON GLOMAR CHALLENGER, LEG $76^{1}$
}

\author{
Robert E. Sheridan, Department of Geology, University of Delaware, Newark, Delaware
}

\section{DATA COLLECTION AND PRESENTATION}

Leg 76 began in Norfolk, Virginia, on 11 October and returned to port in Fort Lauderdale, Florida for engine repairs on 23 October, for a crew change on 1 December, and finally for the termination of the leg on 21 December 1980. The track made by Glomar Challenger during this cruise is shown in Figure 1. Position along the track in Greenwich Mean Time (GMT) is shown progressively by the small numbers, and the completed drill sites are indicated. Positioning information is listed in Table 1 along with calculated course and speed maintained between fixes. Navigation was achieved with satellite fixes supplemented with Loran A and C when available. Accuracy of these underway positions is usually within one nautical mile, and the final refined position on station is accurate to within a few tenths of a nautical mile (Talwani et al., 1966). The regional magnetic anomaly for each navigation point was calculated from the coefficients of Cain et al. (1968), and these values were used to compute the magnetic anomaly profiles shown in Figures 2 and 3.

The magnetic anomaly and bathymetric data are presented as profiles (Fig. 3) plotted against linear distance along the track, as indicated on the horizontal scale given in hundreds of nautical miles. Also plotted in digital form are the latitude and longitude at navigation points, date, and time. The vertical scales in Figure 3 are given in gammas for the magnetic anomaly profile and in uncorrected meters for water depth. The magnetic data are the upper of the two profiles and have less variation. A nominal speed of sound of $1500 \mathrm{~m} / \mathrm{s}$ is assumed to calculate the bathymetric data, and the presentation of the profiles follow the format of the Deep Sea Drilling Project, which kindly provided these illustrations. The locations of the drill sites completed are also indicated on the plots.

The continuous seismic reflection profile is shown in Figure 4 (back pocket of this volume). Reflection times in seconds of two-way traveltime are plotted along the sides of the profile, and they can be used to determine depth at a conversion rate of $750 \mathrm{~m} / \mathrm{s}$. The tick marks along the bottom of the profile mark half-hour intervals; course and speed changes are also marked. The time (given in Greenwich Mean Time), date, speed, and heading maintained between course and speed changes are also indicated. Portions of the profiler record discussed

'Sheridan, R. E., Gradstein, F. M., et al., Init. Repts. DSDP, 76: Washington (U.S. Govt. Printing Office). in the text are cited by time and date. The sites are noted along the upper edge of the profiles. Individual profiles can be located along the track (Fig. 1) by converting GMT (ship's time) to location and distance along the track using Table 1.

Two Bolt air guns fired every $20 \mathrm{~s}$ at 2000 psi provided the sound source for these profiles. Chamber sizes were 150 in. ${ }^{3}$ for one gun and 40 in. ${ }^{3}$ for the second gun. Filter settings were generally 20 to $160 \mathrm{~Hz}$. The data presented here were recorded on two recorders, one at a 10 -s (EDO \#1) and one at a 2.5 -s (EDO \#2) sweep.

\section{DISCUSSION}

The underway geophysical gear was streamed shortly after Glomar Challenger left Norfolk, Virginia, and had reached water sufficiently deep to tow the array. We obtained a good profile transverse to the continental margin. Rough steep topography of the canyon-dissected continental slope gives way southeastward to the smooth apron of the continental rise sedimentary prism (Figs. 3 and 4,12 October). The continental rise levels off markedly at $0900 \mathrm{Z}, 12$ October 1980 , where $0.5 \mathrm{~s}$ of well-stratified turbitites overlie what might be a gas hydrate bottom-simulating reflector (BSR) at $1.0 \mathrm{~s}$ sub-bottom depth. None of the deeper reflectors of $\mathrm{A}^{\mathrm{u}}, \mathrm{A}^{*}, \beta$, or oceanic basement appear, because the relatively low energy of Glomar Challenger's air guns failed to penetrate the 1.0 $\mathrm{s}$ of sedimentary section. First appearance of the flank and bedding structure of the Blake Outer Ridge occurs at $0200 \mathrm{Z}, 17$ October 1980 (Fig. 4). Site 533 was drilled here on the crest of the Blake Outer Ridge.

The magnetometer data underway to Site 533 are characterized by low-amplitude, 50-gamma anomalies typical of the Jurassic magnetic quiet zone. A prominent anomaly at 25 nautical miles (n. mi.) is an outer branch of the East Coast Magnetic Anomaly (ECMA) (Taylor et al., 1968; Rabinowitz, 1974).

After leaving Site 533, Glomar Challenger sailed south toward the Blake-Bahama Basin. This track (Fig. 1) parallels the strike of the small-amplitude, 50-gamma magnetic anomalies of the magnetic quiet zone (Fig. 2 and 3 ), and consequently the profile is remarkably smooth. The water depths of the area of the southwest flank of the Blake Outer Ridge are clearly recorded on this track and are most revealing. As Glomar Challenger approached the Blake-Bahama Basin Site 534 from the north, its track crossed the southern part of the Blake Outer Ridge and the ancillary ridge crest forming on its southwest flank between $0900 \mathrm{Z}$ and $1800 \mathrm{Z}, 20$ October 1980 (Figs. 3 and 4). Horizon $A^{u}$, which passes beneath 


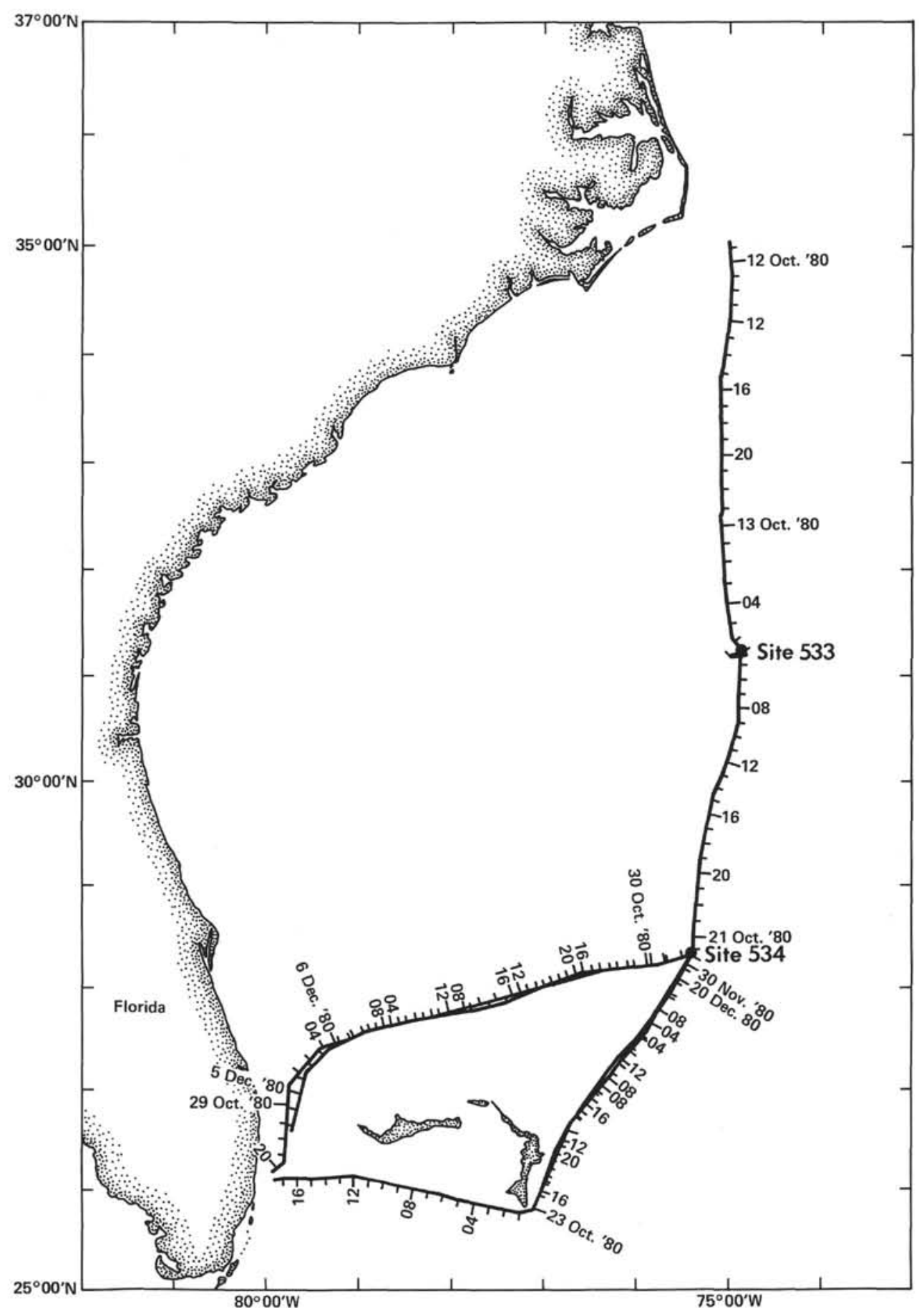

Figure 1. Track chart of Glomar Challenger during Leg 76 from Norfolk, Virginia, to Fort Lauderdale, Florida. (Locations of Sites 533 and 534 are indicated. Smaller numbers denote dates and GMT shiptime along track in hours.)

the ridge, is very weak on these profiles, but the details of bedding structures that cross the bottom-simulating reflector of clathrate origin are clearly visible. The ancillary ridge southwest of the main Blake Outer Ridge crest apparently is forming by current-deposited sediments ponding on its lower flanks (Fig. 4, 1600Z, 20 October 1980).

The "hilly" topography and internal structures of the Blake Outer Ridge are confined to sediments well above horizons $\mathrm{A}^{\mathrm{u}}$ and $\mathrm{X}$ (Fig. 4). The deeper reflectors $\mathrm{X}$ and $\mathrm{A}^{\mathrm{u}}$, which we also see along this track for the first time at this position $(1600 \mathrm{Z})$ at 1.0 and $1.5 \mathrm{~s}$ sub-bottom, respectively, show little structural relief. The internal structure of these ridges and their consistent wavelength within any one cluster (Figs. 3 and 4) support the conclusion that they originated as current-built mud ridges, as suggested by Heezen and Hollister (1971). Formation by rotation of local slump blocks, as proposed by Bal- 
Table 1. Navigation data for the underway portion of Leg 76 of Glomar Challenger.

\begin{tabular}{|c|c|c|c|c|c|c|c|c|c|c|}
\hline \multirow[b]{2}{*}{ Day } & \multirow[b]{2}{*}{ Month } & \multirow[b]{2}{*}{ Year } & \multirow[b]{2}{*}{$\begin{array}{l}\text { Time } \\
\text { (GMT) }\end{array}$} & \multirow[b]{2}{*}{$\begin{array}{c}\text { Latitude } \\
\text { (north) }\end{array}$} & \multirow[b]{2}{*}{$\begin{array}{l}\text { Longitude } \\
\text { (west) }\end{array}$} & \multirow[b]{2}{*}{$\begin{array}{c}\text { Distance } \\
\text { (n. mi.) }\end{array}$} & \multirow[b]{2}{*}{$\begin{array}{c}\text { Speed } \\
\text { (knots) }\end{array}$} & \multirow{2}{*}{$\begin{array}{c}\text { Course } \\
\left({ }^{\circ}\right)\end{array}$} & & rift \\
\hline & & & & & & & & & $\begin{array}{l}\text { Speed } \\
\text { (knots) }\end{array}$ & $\begin{array}{c}\text { Heading } \\
\left({ }^{\circ}\right)\end{array}$ \\
\hline 12 & 10 & 1980 & $0622^{\mathrm{a}}$ & $35^{\circ} 2.95^{\prime}$ & $\begin{array}{ll}75^{\circ} & 0.97^{\prime}\end{array}$ & 0.0 & 4.5 & 160 & 3.0 & 52 \\
\hline 12 & 10 & 1980 & 0626 & $35^{\circ} 2.7^{\prime}$ & $75^{\circ} \quad 0.8^{\prime}$ & 0.3 & 7.7 & 172 & 3.0 & 52 \\
\hline 12 & 10 & 1980 & 0646 & $35^{\circ} 0.1^{\prime}$ & $75^{\circ} 0.4^{\prime}$ & 2.9 & 7.6 & 174 & 3.0 & 52 \\
\hline 12 & 10 & 1980 & $0856^{\mathrm{a}}$ & $34^{\circ} 43.67^{\prime}$ & $74^{\circ} 58.48^{\prime}$ & 19.4 & 8.0 & 184 & 1.8 & 38 \\
\hline 12 & 10 & 1980 & $1040^{\mathrm{a}}$ & $34^{\circ} 29.76^{\prime}$ & $74^{\circ} 59.62^{\prime}$ & 33.3 & 8.9 & 180 & 1.7 & 72 \\
\hline 12 & 10 & 1980 & $1130^{\mathrm{a}}$ & $34^{\circ} 22.32^{\prime}$ & $74^{\circ} 59.63^{\prime}$ & 40.8 & 9.1 & 186 & 0.8 & 63 \\
\hline 12 & 10 & 1980 & $1304^{\mathrm{a}}$ & $34^{\circ} 8.10^{\prime}$ & $75^{\circ} 1.36^{\prime}$ & 55.1 & 9.6 & 189 & 0.2 & 106 \\
\hline 12 & 10 & 1980 & $1450^{\mathrm{a}}$ & $33^{\circ} 51.29^{\prime}$ & $75^{\circ} \quad 4.47^{\prime}$ & 72.1 & 10.0 & 194 & 0.8 & 255 \\
\hline 12 & 10 & 1980 & 1512 & $33^{\circ} 47.8^{\prime}$ & $75^{\circ} 5.5^{\prime}$ & 75.7 & 9.8 & 184 & 0.8 & 255 \\
\hline 12 & 10 & 1980 & $1556^{\mathrm{a}}$ & $33^{\circ} 40.57^{\prime}$ & $75^{\circ} 6.21^{\prime}$ & 82.9 & 9.3 & 178 & 0.4 & 39 \\
\hline 12 & 10 & 1980 & 1617 & $33^{\circ} 37.3^{\prime}$ & $75^{\circ} 6.1^{\prime}$ & 86.2 & 9.3 & 176 & 0.4 & 39 \\
\hline 12 & 10 & 1980 & $1744^{a}$ & $33^{\circ} 23.90^{\prime}$ & $75^{\circ} 5.05^{\prime}$ & 99.6 & 9.6 & 184 & 1.0 & 268 \\
\hline 12 & 10 & 1980 & 1809 & $33^{\circ} 19.9^{\prime}$ & $75^{\circ} 5.4^{\prime}$ & 103.7 & 9.6 & 183 & 1.0 & 268 \\
\hline 12 & 10 & 1980 & $1820^{\mathrm{a}}$ & $33^{\circ} 18.12^{\prime}$ & $75^{\circ} \quad 5.49^{\prime}$ & 105.4 & 8.6 & 181 & 1.1 & 328 \\
\hline 12 & 10 & 1980 & $2050^{\mathrm{a}}$ & $32^{\circ} 56.54^{\prime}$ & $75^{\circ} 5.80^{\prime}$ & 127.0 & 10.5 & 178 & 0.9 & 191 \\
\hline 12 & 10 & 1980 & 2123 & $32^{\circ} 50.8^{\prime}$ & $75^{\circ} \quad 5.6^{\prime}$ & 132.8 & 10.5 & 176 & 0.9 & 191 \\
\hline 12 & 10 & 1980 & $2236^{\mathrm{a}}$ & $32^{\circ} 38.00^{\prime}$ & $75^{\circ} 4.64^{\prime}$ & 145.6 & 10.0 & 177 & 0.5 & 224 \\
\hline 12 & 10 & 1980 & 2310 & $32^{\circ} 32.4^{\prime}$ & $75^{\circ} \quad 4.3^{\prime}$ & 151.2 & 10.1 & 196 & 0.5 & 224 \\
\hline 12 & 10 & 1980 & 2316 & $32^{\circ} 31.4^{\prime}$ & $75^{\circ} 4.7^{\prime}$ & 152.2 & 10.1 & 206 & 0.5 & 224 \\
\hline 12 & 10 & 1980 & 2326 & $32^{\circ} 29.9^{\prime}$ & $75^{\circ} 5.5^{\prime}$ & 153.9 & 10.0 & 177 & 0.5 & 224 \\
\hline 13 & 10 & 1980 & $0018^{a}$ & $32^{\circ} 21.24^{\prime}$ & $75^{\circ} 5.07^{\prime}$ & 162.6 & 10.5 & 178 & 1.0 & 204 \\
\hline 13 & 10 & 1980 & 0050 & $32^{\circ} 15.7^{\prime}$ & $75^{\circ} \quad 4.8^{\prime}$ & 168.1 & 10.4 & 175 & 1.0 & 204 \\
\hline 13 & 10 & 1980 & $0204^{\mathrm{a}}$ & $32^{\circ} 2.85^{\prime}$ & $75^{\circ} 3.42^{\prime}$ & 181.0 & 10.8 & 179 & 1.7 & 219 \\
\hline 13 & 10 & 1980 & $0310^{\mathrm{a}}$ & $31^{\circ} 50.96^{\prime}$ & $75^{\circ} 3.07^{\prime}$ & 192.9 & 11.4 & 173 & 1.8 & 179 \\
\hline 13 & 10 & 1980 & 0338 & $31^{\circ} 45.7^{\prime}$ & $75^{\circ} \quad 2.3^{\prime}$ & 198.2 & 11.4 & 169 & 1.8 & 179 \\
\hline 13 & 10 & 1980 & $0354^{a}$ & $31^{\circ} 42.68^{\prime}$ & $75^{\circ} 1.63^{\prime}$ & 201.3 & 11.5 & 171 & 2.0 & 189 \\
\hline 13 & 10 & 1980 & 0539 & $31^{\circ} 22.8^{\prime}$ & $74^{\circ} 57.8^{\prime}$ & 221.4 & 11.6 & 182 & 2.0 & 189 \\
\hline 13 & 10 & 1980 & 0546 & $31^{\circ} 21.5^{\prime}$ & $74^{\circ} 57.9^{\prime}$ & 222.7 & 8.6 & 142 & 2.0 & 189 \\
\hline 13 & 10 & 1980 & 0555 & $31^{\circ} 20.5^{\prime}$ & $74^{\circ} 57.0^{\prime}$ & 224.0 & 7.9 & 138 & 2.0 & 189 \\
\hline 13 & 10 & 1980 & $0720^{\mathrm{a}}$ & $31^{\circ} 12.14^{\prime}$ & $74^{\circ} 48.24^{\prime}$ & 235.2 & 7.3 & 127 & 0.6 & 154 \\
\hline 13 & 10 & 1980 & 0724 & $31^{\circ} 11.8^{\prime}$ & $74^{\circ} 47.8^{\prime}$ & 235.7 & 6.6 & 265 & 0.6 & 154 \\
\hline 13 & 10 & 1980 & 0856 & $31^{\circ} 11.0^{\prime}$ & $74^{\circ} 59.5^{\prime}$ & 245.7 & 6.7 & 52 & 0.6 & 154 \\
\hline 13 & 10 & 1980 & $0906^{a}$ & $31^{\circ} 11.72^{\prime}$ & $74^{\circ} 58.48^{\prime}$ & 246.9 & 6.7 & 63 & 1.9 & 147 \\
\hline 13 & 10 & 1980 & 0928 & $31^{\circ} 12.8^{\prime}$ & $74^{\circ} 55.9^{\prime}$ & 249.3 & 7.0 & 71 & 1.9 & 147 \\
\hline 13 & 10 & 1980 & 1011 & $31^{\circ} 14.4^{\prime}$ & $74^{\circ} 50.4^{\prime}$ & 254.3 & 6.3 & 244 & 1.9 & 147 \\
\hline 13 & 10 & 1980 & 1020 & $31^{\circ} 14.0^{\prime}$ & $74^{\circ} 51.4^{\prime}$ & 255.3 & 5.2 & 290 & 1.9 & 147 \\
\hline 13 & 10 & 1980 & 1021 & $31^{\circ} 14.1^{\prime}$ & $74^{\circ} 51.4^{\prime}$ & 255.4 & 4.9 & 345 & 1.9 & 147 \\
\hline 13 & 10 & 1980 & 1025 & $31^{\circ} 14.4^{\prime}$ & $74^{\circ} 51.5^{\prime}$ & 255.7 & 4.9 & 317 & 1.9 & 147 \\
\hline 13 & 10 & 1980 & 1042 & $31^{\circ} 15.4^{\prime}$ & $74^{\circ} 52.7^{\prime}$ & 257.1 & 6.7 & 61 & 1.9 & 147 \\
\hline 13 & 10 & 1980 & 1045 & $31^{\circ} 15.5^{\prime}$ & $74^{\circ} 52.3^{\prime}$ & 257.4 & 6.7 & 61 & 1.9 & 147 \\
\hline 13 & 10 & 1980 & $1046^{\mathrm{a}}$ & $31^{\circ} 15.60^{\prime}$ & $74^{\circ} 52.19^{\prime}$ & 257.5 & 6.8 & 45 & 0.0 & 0 \\
\hline 13 & 10 & 1980 & 1046 & $31^{\circ} 15.6^{\prime}$ & $74^{\circ} 52.2^{\prime}$ & 257.5 & 0.0 & 0 & 0.0 & 0 \\
\hline 20 & 10 & 1980 & $0351^{a}$ & $31^{\circ} 15.62^{\prime}$ & $74^{\circ} 52.19^{\prime}$ & 257.5 & 0.9 & 83 & 0.9 & 83 \\
\hline 20 & 10 & 1980 & 0351 & $31^{\circ} 15.6^{\prime}$ & $74^{\circ} 52.2^{\prime}$ & 257.5 & 6.0 & 182 & 0.9 & 83 \\
\hline 20 & 10 & 1980 & 0412 & $31^{\circ} 13.5^{\prime}$ & $74^{\circ} 52.3^{\prime}$ & 259.6 & 8.8 & 185 & 0.9 & 83 \\
\hline 20 & 10 & 1980 & 0420 & $31^{\circ} 12.4^{\prime}$ & $74^{\circ} 52.4^{\prime}$ & 260.8 & 7.8 & 184 & 0.9 & 83 \\
\hline 20 & 10 & 1980 & 0436 & $31^{\circ} 10.3^{\prime}$ & $74^{\circ} 52.6^{\prime}$ & 262.9 & 7.6 & 184 & 0.9 & 83 \\
\hline 20 & 10 & 1980 & 0449 & $31^{\circ} 8.6^{\prime}$ & $74^{\circ} 52.7^{\prime}$ & 264.5 & 8.8 & 185 & 0.9 & 83 \\
\hline 20 & 10 & 1980 & $0654^{\mathrm{a}}$ & $30^{\circ} 50.41^{\prime}$ & $74^{\circ} 54.44^{\prime}$ & 282.8 & 7.9 & 181 & 1.7 & 56 \\
\hline 20 & 10 & 1980 & $0746^{\mathrm{a}}$ & $30^{\circ} 43.54^{\prime}$ & $74^{\circ} 54.61^{\prime}$ & 289.7 & 8.1 & 179 & 1.9 & 65 \\
\hline 20 & 10 & 1980 & 0826 & $30^{\circ} 38.2^{\prime}$ & $74^{\circ} 54.5^{\prime}$ & 295.1 & 8.0 & 184 & 1.9 & 65 \\
\hline 20 & 10 & 1980 & $0842^{\mathrm{a}}$ & $30^{\circ} 36.05^{\prime}$ & $74^{\circ} 54.68^{\prime}$ & 297.2 & 8.5 & 189 & 0.8 & 68 \\
\hline 20 & 10 & 1980 & 0912 & $30^{\circ} 31.8^{\prime}$ & $74^{\circ} 55.5^{\prime}$ & 301.5 & 8.4 & 198 & 0.8 & 68 \\
\hline 20 & 10 & 1980 & $0926^{a}$ & $30^{\circ} 29.97^{\prime}$ & $74^{\circ} 56.20^{\prime}$ & 303.4 & 7.7 & 193 & 1.8 & 63 \\
\hline 20 & 10 & 1980 & $1112^{\mathrm{a}}$ & $30^{\circ} 16.68^{\prime}$ & $74^{\circ} 59.82^{\prime}$ & 317.1 & 7.4 & 198 & 1.7 & 40 \\
\hline 20 & 10 & 1980 & $1256^{\mathrm{a}}$ & $30^{\circ} \quad 4.42^{\prime}$ & $75^{\circ} \quad 4.43^{\prime}$ & 330.0 & 8.0 & 203 & 1.1 & 13 \\
\hline 20 & 10 & 1980 & $1420^{\mathrm{a}}$ & $29^{\circ} 54.17^{\prime}$ & $75^{\circ} 9.49^{\prime}$ & 341.1 & 8.2 & 201 & 0.8 & 33 \\
\hline 20 & 10 & 1980 & 1440 & $29^{\circ} 51.6^{\prime}$ & $75^{\circ} 10.6^{\prime}$ & 343.9 & 8.2 & 190 & 0.8 & 33 \\
\hline 20 & 10 & 1980 & $1528^{\mathrm{a}}$ & $29^{\circ} 45.12^{\prime}$ & $75^{\circ} 11.94^{\prime}$ & 350.4 & 7.9 & 190 & 1.2 & 23 \\
\hline 20 & 10 & 1980 & $1604^{\mathrm{a}}$ & $29^{\circ} 40.47^{\prime}$ & $75^{\circ} 12.92^{\prime}$ & 355.2 & 8.5 & 191 & 0.5 & 30 \\
\hline 20 & 10 & 1980 & $1846^{\mathrm{a}}$ & $29^{\circ} 17.97^{\prime}$ & $75^{\circ} 17.88^{\prime}$ & 378.1 & 8.9 & 191 & 0.1 & 55 \\
\hline 20 & 10 & 1980 & 1908 & $29^{\circ} 14.8^{\prime}$ & $75^{\circ} 18.6^{\prime}$ & 381.3 & 8.9 & 184 & 0.1 & 55 \\
\hline 20 & 10 & 1980 & $2034^{\mathrm{a}}$ & $29^{\circ} \quad 2.03^{\prime}$ & $75^{\circ} 19.73^{\prime}$ & 394.1 & 9.3 & 185 & 0.3 & 187 \\
\hline 20 & 10 & 1980 & 2238 & $28^{\circ} 42.9^{\prime}$ & $75^{\circ} 21.7^{\prime}$ & 413.3 & 9.3 & 184 & 0.3 & 187 \\
\hline 21 & 10 & 1980 & $0004^{a}$ & $28^{\circ} 29.61^{\prime}$ & $75^{\circ} 22.74^{\prime}$ & 426.7 & 9.5 & 181 & 0.6 & 144 \\
\hline 21 & 10 & 1980 & 0010 & $28^{\circ} 28.7^{\prime}$ & $75^{\circ} 22.8^{\prime}$ & 427.6 & 7.3 & 181 & 0.6 & 144 \\
\hline 21 & 10 & 1980 & $0058^{\mathrm{a}}$ & $28^{\circ} 22.82^{\prime}$ & $75^{\circ} 22.85^{\prime}$ & 433.4 & 8.2 & 182 & 1.4 & 172 \\
\hline
\end{tabular}


Table 1. (Continued).

\begin{tabular}{|c|c|c|c|c|c|c|c|c|c|c|}
\hline \multirow[b]{2}{*}{ Day } & \multirow[b]{2}{*}{ Month } & \multirow[b]{2}{*}{ Year } & \multirow[b]{2}{*}{$\begin{array}{l}\text { Time } \\
\text { (GMT) }\end{array}$} & \multirow[b]{2}{*}{$\begin{array}{l}\text { Latitude } \\
\text { (north) }\end{array}$} & \multirow[b]{2}{*}{$\begin{array}{l}\text { Longitude } \\
\text { (west) }\end{array}$} & \multirow[b]{2}{*}{$\begin{array}{l}\text { Distance } \\
\text { (n. mi.) }\end{array}$} & \multirow[b]{2}{*}{$\begin{array}{c}\text { Speed } \\
\text { (knots) }\end{array}$} & \multirow[b]{2}{*}{$\begin{array}{c}\text { Course } \\
\left({ }^{\circ}\right)\end{array}$} & & ift \\
\hline & & & & & & & & & $\begin{array}{l}\text { Speed } \\
\text { (knots) }\end{array}$ & $\begin{array}{c}\text { Heading } \\
\left({ }^{\circ}\right)\end{array}$ \\
\hline 21 & 10 & 1980 & $0114^{a}$ & $28^{\circ} 20.63^{\prime}$ & $75^{\circ} 22.93^{\prime}$ & 435.6 & 6.8 & 184 & 0.0 & 0 \\
\hline 21 & 10 & 1980 & 0114 & $28^{\circ} 20.6^{\prime}$ & $75^{\circ} 22.9^{\prime}$ & 435.6 & 0.0 & 0 & 0.0 & 0 \\
\hline 22. & 10 & 1980 & $0342^{\mathrm{a}}$ & $28^{\circ} 20.63^{\prime}$ & $75^{\circ} 22.93^{\prime}$ & 435.6 & 1.7 & 59 & 1.7 & 59 \\
\hline 22 & 10 & 1980 & 0342 & $28^{\circ} 20.6^{\prime}$ & $75^{\circ} 22.9^{\prime}$ & 435.6 & 4.7 & 205 & 1.7 & 59 \\
\hline 22 & 10 & 1980 & 0355 & $28^{\circ} 19.7^{\prime}$ & $75^{\circ} 23.4^{\prime}$ & 436.7 & 7.5 & 209 & 1.7 & 59 \\
\hline 22 & 10 & 1980 & $0414^{a}$ & $28^{\circ} 17.61^{\prime}$ & $75^{\circ} 24.72^{\prime}$ & 439.0 & 9.2 & 209 & 0.8 & 133 \\
\hline 22 & 10 & 1980 & $0702^{\mathrm{a}}$ & $27^{\circ} 55.18^{\prime}$ & $75^{\circ} 38.82^{\prime}$ & 464.7 & 9.1 & 211 & 0.5 & 134 \\
\hline 22 & 10 & 1980 & 0734 & $27^{\circ} 51.0^{\prime}$ & $75^{\circ} 41.6^{\prime}$ & 469.5 & 9.1 & 215 & 0.5 & 134 \\
\hline 22 & 10 & 1980 & $0750^{\mathrm{a}}$ & $27^{\circ} 49.04^{\prime}$ & $75^{\circ} 43.21^{\prime}$ & 472.0 & 9.5 & 218 & 0.6 & 224 \\
\hline 22 & 10 & 1980 & $0850^{\mathrm{a}}$ & $27^{\circ} 41.55^{\prime}$ & $75^{\circ} 49.90^{\prime}$ & 481.5 & 9.4 & 219 & 0.4 & 238 \\
\hline 22 & 10 & 1980 & $0936^{\mathrm{a}}$ & $27^{\circ} 35.94^{\prime}$ & $75^{\circ} 55.01^{\prime}$ & 488.7 & 8.9 & 220 & 0.3 & 327 \\
\hline 22 & 10 & 1980 & $1204^{\mathrm{a}}$ & $27^{\circ} 19.16^{\prime}$ & $76^{\circ} 10.94^{\prime}$ & 510.7 & 8.4 & 221 & 0.7 & 2 \\
\hline 22 & 10 & 1980 & 1222 & $27^{\circ} 17.2^{\prime}$ & $76^{\circ} 12.8^{\prime}$ & 513.2 & 8.4 & 216 & 0.7 & 2 \\
\hline 22 & 10 & 1980 & $1604^{\mathrm{a}}$ & $26^{\circ} 51.98^{\prime}$ & $76^{\circ} 33.07^{\prime}$ & 544.2 & 8.5 & 217 & 0.8 & 349 \\
\hline 22 & 10 & 1980 & 1640 & $26^{\circ} 47.9^{\prime}$ & $76^{\circ} 36.5^{\prime}$ & 549.3 & 8.4 & 213 & 0.8 & 349 \\
\hline 22 & 10 & 1980 & 1734 & $26^{\circ} 41.6^{\prime}$ & $76^{\circ} 41.2^{\prime}$ & 556.9 & 8.4 & 203 & 0.8 & 349 \\
\hline 22 & 10 & 1980 & 1818 & $26^{\circ} 35.9^{\prime}$ & $76^{\circ} 43.8^{\prime}$ & 563.0 & 8.4 & 205 & 0.8 & 349 \\
\hline 22 & 10 & 1980 & 1851 & $26^{\circ} 31.7^{\prime}$ & $76^{\circ} 46.0^{\prime}$ & 567.6 & 8.4 & 204 & 0.8 & 349 \\
\hline 22 & 10 & 1980 & $1854^{\mathrm{a}}$ & $26^{\circ} 31.36^{\prime}$ & $76^{\circ} 46.16^{\prime}$ & 568.1 & 8.6 & 202 & 0.5 & 5 \\
\hline 22 & 10 & 1980 & 1935 & $26^{\circ} 25.9^{\prime}$ & $76^{\circ} 48.6^{\prime}$ & 573.9 & 8.6 & 204 & 0.5 & 5 \\
\hline 22 & 10 & 1980 & $2042^{\mathrm{a}}$ & $26^{\circ} 17.18^{\prime}$ & $76^{\circ} 52.92^{\prime}$ & 583.5 & 9.2 & 204 & 0.3 & 239 \\
\hline 22 & 10 & 1980 & 2313 & $25^{\circ} 56.0^{\prime}$ & $77^{\circ} 3.4^{\prime}$ & 606.7 & 9.2 & 201 & 0.3 & 239 \\
\hline 22 & 10 & 1980 & $2334^{\mathrm{a}}$ & $25^{\circ} 52.96^{\prime}$ & $77^{\circ} 4.74^{\prime}$ & 609.9 & 9.3 & 199 & 0.4 & 178 \\
\hline 22 & 10 & 1980 & 2354 & $25^{\circ} 50.0^{\prime}$ & $77^{\circ} 5.9^{\prime}$ & 613.0 & 7.1 & 199 & 0.4 & 178 \\
\hline 22 & 10 & 1980 & 2359 & $25^{\circ} 49.5^{\prime}$ & $77^{\circ} \quad 6.1^{\prime}$ & 613.6 & 9.3 & 199 & 0.4 & 178 \\
\hline 23 & 10 & 1980 & 0003 & $25^{\circ} 48.9^{\prime}$ & $77^{\circ} \quad 6.3^{\prime}$ & 614.3 & 9.3 & 202 & 0.4 & 178 \\
\hline 23 & 10 & 1980 & 0012 & $25^{\circ} 47.6^{\prime}$ & $77^{\circ} \quad 6.9^{\prime}$ & 615.7 & 9.1 & 258 & 0.4 & 178 \\
\hline 23 & 10 & 1980 & 0039 & $25^{\circ} 46.7^{\prime}$ & $77^{\circ} 11.3^{\prime}$ & 619.7 & 6.9 & 257 & 0.4 & 178 \\
\hline 23 & 10 & 1980 & 0046 & $25^{\circ} 46.5^{\prime}$ & $77^{\circ} 12.2^{\prime}$ & 620.5 & 9.1 & 258 & 0.4 & 178 \\
\hline 23 & 10 & 1980 & 0054 & $25^{\circ} 46.3^{\prime}$ & $77^{\circ} 13.5^{\prime}$ & 621.7 & 8.9 & 280 & 0.4 & 178 \\
\hline 23 & 10 & 1980 & $0108^{a}$ & $25^{\circ} 46.62^{\prime}$ & $77^{\circ} 15.77^{\prime}$ & 623.8 & 9.3 & 283 & 0.4 & 309 \\
\hline 23 & 10 & 1980 & 0224 & $25^{\circ} 49.3^{\prime}$ & $77^{\circ} 28.6^{\prime}$ & 635.7 & 9.1 & 283 & 0.4 & 309 \\
\hline 23 & 10 & 1980 & $0254^{\mathrm{a}}$ & $25^{\circ} 50.34^{\prime}$ & $77^{\circ} 33.53^{\prime}$ & 640.2 & 9.2 & 284 & 0.5 & 323 \\
\hline 23 & 10 & 1980 & $0324^{\mathrm{a}}$ & $25^{\circ} 51.44^{\prime}$ & $77^{\circ} 38.47^{\prime}$ & 644.8 & 9.3 & 282 & 0.5 & 285 \\
\hline 23 & 10 & 1980 & 0506 & $25^{\circ} 54.8^{\prime}$ & $77^{\circ} 55.7^{\prime}$ & 660.6 & 9.3 & 283 & 0.5 & 285 \\
\hline 23 & 10 & 1980 & $0512^{\mathrm{a}}$ & $25^{\circ} 54.99^{\prime}$ & $77^{\circ} 56.66^{\prime}$ & 661.6 & 9.6 & 288 & 1.1 & 332 \\
\hline 23 & 10 & 1980 & $0612^{\mathrm{a}}$ & $25^{\circ} 57.96^{\prime}$ & $78^{\circ} 6.79^{\prime}$ & 671.1 & 8.8 & 281 & 0.3 & 187 \\
\hline 23 & 10 & 1980 & $0800^{\mathrm{a}}$ & $26^{\circ} 0.95^{\prime}$ & $78^{\circ} 24.05^{\prime}$ & 686.9 & 8.9 & 283 & 0.1 & 261 \\
\hline 23 & 10 & 1980 & $0848^{\mathrm{a}}$ & $26^{\circ} 2.52^{\prime}$ & $78^{\circ} 31.77^{\prime}$ & 694.0 & 8.7 & 282 & 0.1 & 161 \\
\hline 23 & 10 & 1980 & $1036^{\mathrm{a}}$ & $26^{\circ} 5.90^{\prime}$ & $78^{\circ} 48.88^{\prime}$ & 709.8 & 8.5 & 283 & 0.3 & 115 \\
\hline 23 & 10 & 1980 & $1118^{\mathrm{a}}$ & $26^{\circ} 7.20^{\prime}$ & $78^{\circ} 55.36^{\prime}$ & 715.7 & 8.7 & 290 & 1.1 & 21 \\
\hline 23 & 10 & 1980 & 1150 & $26^{\circ} 8.8^{\prime}$ & $79^{\circ} \quad 0.2^{\prime}$ & 720.4 & 8.4 & 274 & 1.1 & 21 \\
\hline 23 & 10 & 1980 & 1224 & $26^{\circ} 9.1^{\prime}$ & $79^{\circ} 5.5^{\prime}$ & 725.2 & 8.3 & 263 & 1.1 & 21 \\
\hline 23 & 10 & 1980 & $1446^{\mathrm{a}}$ & $26^{\circ} 6.78^{\prime}$ & $79^{\circ} 27.15^{\prime}$ & 744.7 & 8.3 & 270 & 1.9 & 7 \\
\hline 23 & 10 & 1980 & 1644 & $26^{\circ} 6.7^{\prime}$ & $79^{\circ} 45.4^{\prime}$ & 761.1 & 8.2 & 264 & 1.9 & 7 \\
\hline 23 & 10 & 1980 & 1703 & $26^{\circ} \quad 6.4^{\prime}$ & $79^{\circ} 48.3^{\prime}$ & 763.7 & 8.0 & 260 & 1.9 & 7 \\
\hline 23 & 10 & 1980 & $1744^{\mathrm{a}}$ & $26^{\circ} 5.50^{\prime}$ & $79^{\circ} 54.30^{\prime}$ & 769.2 & 8.8 & 248 & 0.0 & 355 \\
\hline 23 & 10 & 1980 & 1744 & $26^{\circ} \quad 5.5^{\prime}$ & $79^{\circ} 54.3^{\prime}$ & 769.2 & 0.0 & 355 & 0.0 & 355 \\
\hline 28 & 10 & 1980 & $1915^{\mathrm{a}}$ & $26^{\circ} 10.20^{\prime}$ & $79^{\circ} 54.80^{\prime}$ & 773.9 & 2.8 & 309 & 2.8 & 309 \\
\hline 28 & 10 & 1980 & 1915 & $26^{\circ} 10.2^{\prime}$ & $79^{\circ} 54.8^{\prime}$ & 773.9 & 5.3 & 43 & 2.8 & 309 \\
\hline 28 & 10 & 1980 & 1930 & $26^{\circ} 11.2^{\prime}$ & $79^{\circ} 53.8^{\prime}$ & 775.2 & 6.4 & 48 & 2.8 & 309 \\
\hline 28 & 10 & 1980 & $2008^{\mathrm{a}}$ & $26^{\circ} 13.92^{\prime}$ & $79^{\circ} 50.44^{\prime}$ & 779.3 & 9.0 & 56 & 2.5 & 13 \\
\hline 28 & 10 & 1980 & 2030 & $26^{\circ} 15.8^{\prime}$ & $79^{\circ} 47.4^{\prime}$ & 782.6 & 9.9 & 8 & 2.5 & 13 \\
\hline 28 & 10 & 1980 & $2154^{\mathrm{a}}$ & $26^{\circ} 29.54^{\prime}$ & $79^{\circ} 45.27^{\prime}$ & 796.5 & 9.8 & 2 & 2.4 & 351 \\
\hline 28 & 10 & 1980 & 2304 & $26^{\circ} 40.9^{\prime}$ & $79^{\circ} 44.8^{\prime}$ & 807.9 & 11.0 & 3 & 2.4 & 351 \\
\hline 29 & 10 & 1980 & 0104 & $27^{\circ} 2.9^{\prime}$ & $79^{\circ} 43.6^{\prime}$ & 829.9 & 10.1 & 39 & 2.4 & 351 \\
\hline 29 & 10 & 1980 & 0342 & $27^{\circ} 23.5^{\prime}$ & $79^{\circ} 25.0^{\prime}$ & 856.3 & 9.2 & 60 & 2.4 & 351 \\
\hline 29 & 10 & 1980 & 0354 & $27^{\circ} 24.4^{\prime}$ & $79^{\circ} 23.2^{\prime}$ & 858.2 & 9.8 & 61 & 2.4 & 351 \\
\hline 29 & 10 & 1980 & 0411 & $27^{\circ} 25.8^{\prime}$ & $79^{\circ} 20.5^{\prime}$ & 860.9 & 10.1 & 61 & 2.4 & 351 \\
\hline 29 & 10 & 1980 & 0417 & $27^{\circ} 26.3^{\prime}$ & $79^{\circ} 19.5^{\prime}$ & 862.0 & 9.4 & 75 & 2.4 & 351 \\
\hline 29 & 10 & 1980 & 0439 & $27^{\circ} 27.1^{\prime}$ & $79^{\circ} 15.7^{\prime}$ & 865.4 & 9.2 & 80 & 2.4 & 351 \\
\hline 29 & 10 & 1980 & 0525 & $27^{\circ} 28.4^{\prime}$ & $79^{\circ} 7.9^{\prime}$ & 872.5 & 10.0 & 63 & 2.4 & 351 \\
\hline 29 & 10 & 1980 & $0636^{\mathrm{a}}$ & $27^{\circ} 33.75^{\prime}$ & $78^{\circ} 56.07^{\prime}$ & 884.2 & 8.6 & 79 & 1.0 & 238 \\
\hline 29 & 10 & 1980 & $0726^{\mathrm{a}}$ & $27^{\circ} 35.11^{\prime}$ & $78^{\circ} 48.14^{\prime}$ & 891.4 & 9.4 & 77 & 0.1 & 227 \\
\hline 29 & 10 & 1980 & $0824^{\mathrm{a}}$ & $27^{\circ} 37.11^{\prime}$ & $78^{\circ} 38.12^{\prime}$ & 900.5 & 9.5 & 74 & 0.5 & 340 \\
\hline 29 & 10 & 1980 & $0912^{\mathrm{a}}$ & $27^{\circ} 39.16^{\prime}$ & $78^{\circ} 29.90^{\prime}$ & 908.1 & 9.6 & 76 & 0.3 & 10 \\
\hline 29 & 10 & 1980 & $1132^{\mathrm{a}}$ & $27^{\circ} 44.77^{\prime}$ & $78^{\circ} 5.37^{\prime}$ & 930.5 & 9.4 & 76 & 0.2 & 319 \\
\hline
\end{tabular}


Table 1. (Continued).

\begin{tabular}{|c|c|c|c|c|c|c|c|c|c|c|}
\hline \multirow[b]{2}{*}{ Day } & \multirow[b]{2}{*}{ Month } & \multirow[b]{2}{*}{ Year } & \multirow[b]{2}{*}{$\begin{array}{l}\text { Time } \\
\text { (GMT) }\end{array}$} & \multirow[b]{2}{*}{$\begin{array}{l}\text { Latitude } \\
\text { (north) }\end{array}$} & \multirow[b]{2}{*}{$\begin{array}{l}\text { Longitude } \\
\text { (west) }\end{array}$} & \multirow[b]{2}{*}{$\begin{array}{l}\text { Distance } \\
\text { (n. mi.) }\end{array}$} & \multirow[b]{2}{*}{$\begin{array}{l}\text { Speed } \\
\text { (knots) }\end{array}$} & \multirow[b]{2}{*}{$\begin{array}{c}\text { Course } \\
\left({ }^{\circ}\right)\end{array}$} & & rift \\
\hline & & & & & & & & & $\begin{array}{l}\text { Speed } \\
\text { (knots) }\end{array}$ & $\begin{array}{c}\text { Heading } \\
\left({ }^{\circ}\right)\end{array}$ \\
\hline 29 & 10 & 1980 & $1318^{\mathrm{a}}$ & $27^{\circ} 48.85^{\prime}$ & $77^{\circ} 47.19^{\prime}$ & 947.1 & 9.4 & 74 & 0.4 & 339 \\
\hline 29 & 10 & 1980 & $1830^{\mathrm{a}}$ & $28^{\circ} 2.07^{\prime}$ & $76^{\circ} 53.65^{\prime}$ & 996.2 & 9.9 & 72 & 0.9 & 14 \\
\hline 29 & 10 & 1980 & 1855 & $28^{\circ} \quad 3.3^{\prime}$ & $76^{\circ} 49.2^{\prime}$ & 1000.3 & 9.9 & 74 & 0.9 & 14 \\
\hline 29 & 10 & 1980 & $1920^{\mathrm{a}}$ & $28^{\circ} 4.45^{\prime}$ & $76^{\circ} 44.67^{\prime}$ & 1004.5 & 9.7 & 74 & 0.8 & 4 \\
\hline 29 & 10 & 1980 & $2016^{\mathrm{a}}$ & $28^{\circ} 6.91^{\prime}$ & $76^{\circ} 34.74^{\prime}$ & 1013.6 & 9.8 & 76 & 0.5 & 18 \\
\hline 29 & 10 & 1980 & $2104^{a}$ & $28^{\circ} 8.74^{\prime}$ & $76^{\circ} 26.14^{\prime}$ & 1021.4 & 10.0 & 79 & 0.5 & 71 \\
\hline 29 & 10 & 1980 & $2242^{\mathrm{a}}$ & $28^{\circ} 11.94^{\prime}$ & $76^{\circ} 8.07^{\prime}$ & 1037.6 & 10.9 & 81 & 1.5 & 92 \\
\hline 29 & 10 & 1980 & 2308 & $28^{\circ} 12.7^{\prime}$ & $76^{\circ} 2.8^{\prime}$ & 1042.4 & 10.9 & 82 & 1.5 & 92 \\
\hline 30 & 10 & 1980 & $0026^{\mathrm{a}}$ & $28^{\circ} 14.76^{\prime}$ & $75^{\circ} 46.82^{\prime}$ & 1056.6 & 10.1 & 83 & 0.8 & 118 \\
\hline 30 & 10 & 1980 & $0048^{\mathrm{a}}$ & $28^{\circ} 15.23^{\prime}$ & $75^{\circ} 42.63^{\prime}$ & 1060.3 & 9.9 & 80 & 0.4 & 71 \\
\hline 30 & 10 & 1980 & 0104 & $28^{\circ} 15.7^{\prime}$ & $75^{\circ} 39.7^{\prime}$ & 1062.9 & 9.9 & 75 & 0.4 & 71 \\
\hline 30 & 10 & 1980 & 0133 & $28^{\circ} 16.9^{\prime}$ & $75^{\circ} 34.5^{\prime}$ & 1067.7 & 9.9 & 68 & 0.4 & 71 \\
\hline 30 & 10 & 1980 & 0143 & $28^{\circ} 17.6^{\prime}$ & $75^{\circ} 32.7^{\prime}$ & 1069.3 & 0.4 & 71 & 0.4 & 71 \\
\hline 31 & 10 & 1980 & $0254^{\mathrm{a}}$ & $28^{\circ} 20.63^{\prime}$ & $75^{\circ} 22.89^{\prime}$ & 1078.5 & 0.0 & 0 & 0.0 & 0 \\
\hline 29 & 11 & 1980 & $1130^{\mathrm{a}}$ & $28^{\circ} 20.63^{\prime}$ & $75^{\circ} 22.89^{\prime}$ & 1078.5 & 0.2 & 98 & 0.2 & 98 \\
\hline 29 & 11 & 1980 & 2306 & $28^{\circ} 20.4^{\prime}$ & $75^{\circ} 20.9^{\prime}$ & 1080.3 & 7.3 & 213 & 0.2 & 98 \\
\hline 29 & 11 & 1980 & 2329 & $28^{\circ} 18.0^{\prime}$ & $75^{\circ} 22.6^{\prime}$ & 1083.1 & 9.1 & 213 & 0.2 & 98 \\
\hline 29 & 11 & 1980 & 2346 & $28^{\circ} 15.9^{\prime}$ & $75^{\circ} 24.2^{\prime}$ & 1085.7 & 9.5 & 213 & 0.2 & 98 \\
\hline 30 & 11 & 1980 & $0030^{\mathrm{a}}$ & $28^{\circ} 10.00^{\prime}$ & $75^{\circ} 28.55^{\prime}$ & 1092.7 & 10.1 & 208 & 1.1 & 150 \\
\hline 30 & 11 & 1980 & 0215 & $27^{\circ} 54.4^{\prime}$ & $75^{\circ} 38.1^{\prime}$ & 1110.4 & 10.0 & 214 & 1.1 & 150 \\
\hline 30 & 11 & 1980 & $0220^{\mathrm{a}}$ & $27^{\circ} 53.72^{\prime}$ & $75^{\circ} 38.62^{\prime}$ & 1111.3 & 10.7 & 215 & 1.5 & 176 \\
\hline 30 & 11 & 1980 & 0305 & $27^{\circ} 47.1^{\prime}$ & $75^{\circ} 43.8^{\prime}$ & 1119.3 & 7.7 & 212 & 1.5 & 176 \\
\hline 30 & 11 & 1980 & 0308 & $27^{\circ} 46.8^{\prime}$ & $75^{\circ} 44.0^{\prime}$ & 1119.7 & 9.5 & 214 & 1.5 & 176 \\
\hline 30 & 11 & 1980 & 0322 & $27^{\circ} 44.9^{\prime}$ & $75^{\circ} 45.4^{\prime}$ & 1121.9 & 10.1 & 214 & 1.5 & 176 \\
\hline 30 & 11 & 1980 & $0324^{\mathrm{a}}$ & $27^{\circ} 44.6^{\prime}$ & $75^{\circ} 45.61^{\prime}$ & 1122.2 & 9.7 & 208 & 2.0 & 143 \\
\hline 30 & 11 & 1980 & 0326 & $27^{\circ} 44.4^{\prime}$ & $75^{\circ} 45.8^{\prime}$ & 1122.6 & 10.2 & 209 & 2.0 & 143 \\
\hline 30 & 11 & 1980 & 0424 & $27^{\circ} 35.7^{\prime}$ & $75^{\circ} 51.2^{\prime}$ & 1132.5 & 10.1 & 214 & 2.0 & 143 \\
\hline 30 & 11 & 1980 & $0510^{\mathrm{a}}$ & $27^{\circ} 29.30^{\prime}$ & $75^{\circ} 56.07^{\prime}$ & 1140.2 & 10.5 & 221 & 1.1 & 190 \\
\hline 30 & 11 & 1980 & $062^{\mathrm{a}}$ & $27^{\circ} 22.44^{\prime}$ & $76^{\circ} 2.89^{\prime}$ & 1149.3 & 9.9 & 219 & 1.0 & 151 \\
\hline 30 & 11 & 1980 & $0752^{\mathrm{a}}$ & $27^{\circ} 8.34^{\prime}$ & $76^{\circ} 15.86^{\prime}$ & 1167.6 & 10.1 & 223 & 0.5 & 192 \\
\hline 30 & 11 & 1980 & 0825 & $27^{\circ} 4.3^{\prime}$ & $76^{\circ} 20.1^{\prime}$ & 1173.1 & 10.1 & 220 & 0.5 & 192 \\
\hline 30 & 11 & 1980 & $0846^{\mathrm{a}}$ & $27^{\circ} 1.59^{\prime}$ & $76^{\circ} 22.65^{\prime}$ & 1176.6 & 9.8 & 221 & 0.2 & 237 \\
\hline 30 & 11 & 1980 & $1030^{\mathrm{a}}$ & $26^{\circ} 48.82^{\prime}$ & $76^{\circ} 35.26^{\prime}$ & 1193.6 & 9.9 & 221 & 0.3 & 234 \\
\hline 30 & 11 & 1980 & 1100 & $26^{\circ} 45.1^{\prime}$ & $76^{\circ} 38.9^{\prime}$ & 1198.6 & 9.9 & 218 & 0.3 & 234 \\
\hline 30 & 11 & 1980 & 1148 & $26^{\circ} 38.9^{\prime}$ & $76^{\circ} 44.4^{\prime}$ & 1206.4 & 9.8 & 201 & 0.3 & 234 \\
\hline 30 & 11 & 1980 & 1221 & $26^{\circ} 33.9^{\prime}$ & $76^{\circ} 46.5^{\prime}$ & 1211.8 & 7.6 & 201 & 0.3 & 234 \\
\hline 30 & 11 & 1980 & $1222^{\mathrm{a}}$ & $26^{\circ} 33.78^{\prime}$ & $76^{\circ} 46.59^{\prime}$ & 1212.0 & 8.0 & 202 & 0.6 & 227 \\
\hline 30 & 11 & 1980 & 1227 & $26^{\circ} 33.2^{\prime}$ & $76^{\circ} 46.9^{\prime}$ & 1212.6 & 10.2 & 202 & 0.6 & 227 \\
\hline 30 & 11 & 1980 & 1348 & $26^{\circ} 20.4^{\prime}$ & $76^{\circ} 52.5^{\prime}$ & 1226.3 & 8.0 & 202 & 0.6 & 227 \\
\hline 30 & 11 & 1980 & 1405 & $26^{\circ} 18.3^{\prime}$ & $76^{\circ} 53.4^{\prime}$ & 1228.6 & 10.2 & 202 & 0.6 & 227 \\
\hline 30 & 11 & 1980 & $1412^{\mathrm{a}}$ & $26^{\circ} 17.23^{\prime}$ & $76^{\circ} 53.93^{\prime}$ & 1229.8 & 10.2 & 201 & 0.7 & 213 \\
\hline 30 & 11 & 1980 & $1516^{\mathrm{a}}$ & $26^{\circ} 7.02^{\prime}$ & $76^{\circ} 58.26^{\prime}$ & 1240.7 & 10.3 & 201 & 0.8 & 216 \\
\hline 30 & 11 & 1980 & 1612 & $25^{\circ} 58.0^{\prime}$ & $77^{\circ} 2.1^{\prime}$ & 1250.4 & 10.3 & 197 & 0.8 & 216 \\
\hline 30 & 11 & 1980 & $1700^{\mathrm{a}}$ & $25^{\circ} 50.13^{\prime}$ & $77^{\circ} 4.90^{\prime}$ & 1258.6 & 9.6 & 203 & 1.2 & 288 \\
\hline 30 & 11 & 1980 & 1700 & $25^{\circ} 50.1^{\prime}$ & $77^{\circ} 4.9^{\prime}$ & 1258.6 & 1.2 & 288 & 1.2 & 288 \\
\hline 5 & 12 & 1980 & 1741 & $26^{\circ} 35.9^{\prime}$ & $79^{\circ} 43.1^{\prime}$ & 1407.8 & 6.7 & 9 & 1.2 & 288 \\
\hline 5 & 12 & 1980 & $1742^{\mathrm{a}}$ & $26^{\circ} 36.00^{\prime}$ & $79^{\circ} 42.60^{\prime}$ & 1408.3 & 7.4 & 13 & 1.1 & 329 \\
\hline 5 & 12 & 1980 & 1742 & $26^{\circ} 36.0^{\prime}$ & $79^{\circ} 42.6^{\prime}$ & 1408.3 & 10.0 & 15 & 1.1 & 329 \\
\hline 5 & 12 & 1980 & $1908^{\mathrm{a}}$ & $26^{\circ} 49.80^{\prime}$ & $79^{\circ} 38.50^{\prime}$ & 1422.5 & 9.9 & 13 & 1.4 & 319 \\
\hline 5 & 12 & 1980 & 2012 & $27^{\circ} 0.1^{\prime}$ & $79^{\circ} 35.8^{\prime}$ & 1433.1 & 7.4 & 11 & 1.4 & 319 \\
\hline 5 & 12 & 1980 & 2043 & $27^{\circ} \quad 3.8^{\prime}$ & $79^{\circ} 35.0^{\prime}$ & 1436.9 & 9.9 & 13 & 1.4 & 319 \\
\hline 5 & 12 & 1980 & 2125 & $27^{\circ} 10.6^{\prime}$ & $79^{\circ} 33.2^{\prime}$ & 1443.9 & 9.2 & 44 & 1.4 & 319 \\
\hline 5 & 12 & 1980 & 2323 & $27^{\circ} 23.8^{\prime}$ & $79^{\circ} 19.1^{\prime}$ & 1462.1 & 8.7 & 67 & 1.4 & 319 \\
\hline 6 & 12 & 1980 & 0000 & $27^{\circ} 25.9^{\prime}$ & $79^{\circ} 13.6^{\prime}$ & 1467.4 & 8.7 & 67 & 1.4 & 319 \\
\hline 6 & 12 & 1980 & $0230^{\mathrm{a}}$ & $27^{\circ} 34.40^{\prime}$ & $78^{\circ} 51.00^{\prime}$ & 1489.2 & 7.7 & 77 & 1.5 & 242 \\
\hline 6 & 12 & 1980 & 0300 & $27^{\circ} 35.2^{\prime}$ & $78^{\circ} 46.7^{\prime}$ & 1493.0 & 8.1 & 77 & 1.5 & 242 \\
\hline 6 & 12 & 1980 & $0346^{\mathrm{a}}$ & $27^{\circ} 36.60^{\prime}$ & $78^{\circ} 39.90^{\prime}$ & 1499.2 & 9.4 & 75 & 0.2 & 240 \\
\hline 6 & 12 & 1980 & $0534^{\mathrm{a}}$ & $27^{\circ} 40.90^{\prime}$ & $78^{\circ} 21.40^{\prime}$ & 1516.2 & 9.4 & 80 & 0.9 & 179 \\
\hline 6 & 12 & 1980 & $0900^{\mathrm{a}}$ & $27^{\circ} 46.40^{\prime}$ & $77^{\circ} 45.40^{\prime}$ & 1548.5 & 10.5 & 77 & 1.0 & 99 \\
\hline 6 & 12 & 1980 & 0945 & $27^{\circ} 48.1^{\prime}$ & $77^{\circ} 36.7^{\prime}$ & 1556.4 & 10.5 & 74 & 1.0 & 99 \\
\hline 6 & 12 & 1980 & $1046^{\mathrm{a}}$ & $27^{\circ} 51.00^{\prime}$ & $77^{\circ} 25.10^{\prime}$ & 1567.1 & 9.9 & 69 & 0.6 & 12 \\
\hline 6 & 12 & 1980 & $1106^{\mathrm{a}}$ & $27^{\circ} 52.20^{\prime}$ & $77^{\circ} 21.60^{\prime}$ & 1570.4 & 9.6 & 66 & 1.0 & 337 \\
\hline 6 & 12 & 1980 & 1332 & $28^{\circ} 1.7^{\prime}$ & $76^{\circ} 57.5^{\prime}$ & 1593.7 & 6.6 & 63 & 1.0 & 337 \\
\hline 6 & 12 & 1980 & 1343 & $28^{\circ} \quad 2.2^{\prime}$ & $76^{\circ} 56.3^{\prime}$ & 1594.9 & 9.6 & 66 & 1.0 & 337 \\
\hline 6 & 12 & 1980 & $1420^{a}$ & $28^{\circ} 4.60^{\prime}$ & $76^{\circ} 50.20^{\prime}$ & 1600.8 & 9.3 & 73 & 0.4 & 222 \\
\hline 6 & 12 & 1980 & $1538^{\mathrm{a}}$ & $28^{\circ} 8.10^{\prime}$ & $76^{\circ} 37.10^{\prime}$ & 1612.8 & 9.7 & 76 & 0.7 & 159 \\
\hline 6 & 12 & 1980 & 1620 & $28^{\circ} 9.7^{\prime}$ & $76^{\circ} 29.7^{\prime}$ & 1619.6 & 9.8 & 84 & 0.7 & 159 \\
\hline
\end{tabular}


Table 1. (Continued).

\begin{tabular}{|c|c|c|c|c|c|c|c|c|c|c|}
\hline \multirow[b]{2}{*}{ Day } & \multirow[b]{2}{*}{ Month } & \multirow[b]{2}{*}{ Year } & \multirow[b]{2}{*}{$\begin{array}{l}\text { Time } \\
\text { (GMT) }\end{array}$} & \multirow[b]{2}{*}{$\begin{array}{l}\text { Latitude } \\
\text { (north) }\end{array}$} & \multirow[b]{2}{*}{$\begin{array}{l}\text { Longitude } \\
\text { (west) }\end{array}$} & \multirow[b]{2}{*}{$\begin{array}{l}\text { Distance } \\
\text { (n. mi.) }\end{array}$} & \multirow[b]{2}{*}{$\begin{array}{l}\text { Speed } \\
\text { (knots) }\end{array}$} & \multirow[b]{2}{*}{$\begin{array}{c}\text { Course } \\
\left({ }^{\circ}\right)\end{array}$} & \multicolumn{2}{|c|}{ Drift } \\
\hline & & & & & & & & & $\begin{array}{c}\text { Speed } \\
\text { (knots) }\end{array}$ & $\begin{array}{c}\text { Heading } \\
\left({ }^{\circ}\right)\end{array}$ \\
\hline 6 & 12 & 1980 & 2030 & $28^{\circ} 13.9^{\prime}$ & $75^{\circ} 43.7^{\prime}$ & 1660.3 & 9.6 & 74 & 0.7 & 159 \\
\hline 6 & 12 & 1980 & 2125 & $28^{\circ} 16.3^{\prime}$ & $75^{\circ} 34.1^{\prime}$ & 1669.1 & 6.3 & 76 & 0.7 & 159 \\
\hline 6 & 12 & 1980 & 2137 & $28^{\circ} 16.6^{\prime}$ & $75^{\circ} 32.7^{\prime}$ & 1670.4 & 9.6 & 74 & 0.7 & 159 \\
\hline 6 & 12 & 1980 & 2143 & $28^{\circ} 16.9^{\prime}$ & $75^{\circ} 31.7^{\prime}$ & 1671.3 & 6.3 & 76 & 0.7 & 159 \\
\hline 6 & 12 & 1980 & 2148 & $28^{\circ} 17.0^{\prime}$ & $75^{\circ} 31.1^{\prime}$ & 1671.8 & 9.2 & 74 & 0.7 & 159 \\
\hline 6 & 12 & 1980 & $2155^{\mathrm{a}}$ & $28^{\circ} 17.30^{\prime}$ & $75^{\circ} 29.90^{\prime}$ & 1672.9 & 8.1 & 75 & 1.3 & 220 \\
\hline 6 & 12 & 1980 & $2220^{\mathrm{a}}$ & $28^{\circ} 18.20^{\prime}$ & $75^{\circ} 26.20^{\prime}$ & 1676.3 & 7.5 & 67 & 1.7 & 263 \\
\hline 6 & 12 & 1980 & 2226 & $28^{\circ} 18.5^{\prime}$ & $75^{\circ} 25.4^{\prime}$ & 1677.1 & 4.5 & 65 & 1.7 & 263 \\
\hline 6 & 12 & 1980 & $2248^{\mathrm{a}}$ & $28^{\circ} 19.20^{\prime}$ & $75^{\circ} 23.70^{\prime}$ & 1678.7 & 7.0 & 64 & 1.1 & 27 \\
\hline 6 & 12 & 1980 & 2248 & $28^{\circ} 19.2^{\prime}$ & $75^{\circ} 23.7^{\prime}$ & 1678.7 & 1.1 & 27 & 1.1 & 27 \\
\hline 7 & 12 & 1980 & $0015^{a}$ & $28^{\circ} 20.60^{\prime}$ & $75^{\circ} 22.90^{\prime}$ & 1680.3 & 0.0 & 288 & 0.0 & 288 \\
\hline 19 & 12 & 1980 & 2206 & $28^{\circ} 20.8^{\prime}$ & $75^{\circ} 23.7^{\prime}$ & 1681.0 & 6.6 & 214 & 0.0 & 288 \\
\hline 19 & 12 & 1980 & 2227 & $28^{\circ} 18.9^{\prime}$ & $75^{\circ} 25.1^{\prime}$ & 1683.3 & 9.6 & 214 & 0.0 & 288 \\
\hline 19 & 12 & 1980 & $2310^{\mathrm{a}}$ & $28^{\circ} 13.20^{\prime}$ & $75^{\circ} 29.50^{\prime}$ & 1690.2 & 9.6 & 210 & 0.6 & 120 \\
\hline 20 & 12 & 1980 & 0000 & $28^{\circ} \quad 6.3^{\prime}$ & $75^{\circ} 34.0^{\prime}$ & 1698.2 & 9.6 & 210 & 0.6 & 120 \\
\hline 20 & 12 & 1980 & $0254^{\mathrm{a}}$ & $27^{\circ} 42.30^{\prime}$ & $75^{\circ} 49.80^{\prime}$ & 1725.9 & 9.8 & 217 & 0.5 & 287 \\
\hline 20 & 12 & 1980 & $0446^{a}$ & $27^{\circ} 27.70^{\prime}$ & $76^{\circ} 2.10^{\prime}$ & 1744.1 & 9.2 & 213 & 0.4 & 50 \\
\hline 20 & 12 & 1980 & $0716^{a}$ & $27^{\circ} 8.40^{\prime}$ & $76^{\circ} 16.40^{\prime}$ & 1767.2 & 9.6 & 217 & 0.6 & 308 \\
\hline 20 & 12 & 1980 & $0834^{\mathrm{a}}$ & $26^{\circ} 58.50^{\prime}$ & $76^{\circ} 24.90^{\prime}$ & 1779.7 & 9.5 & 220 & 1.0 & 315 \\
\hline 20 & 12 & 1980 & $0858^{a}$ & $26^{\circ} 55.60^{\prime}$ & $76^{\circ} 27.60^{\prime}$ & 1783.5 & 9.4 & 219 & 0.9 & 320 \\
\hline 20 & 12 & 1980 & $1022^{\mathrm{a}}$ & $26^{\circ} 45.40^{\prime}$ & $76^{\circ} 36.90^{\prime}$ & 1796.6 & 9.8 & 217 & 0.6 & 282 \\
\hline 20 & 12 & 1980 & 1200 & $26^{\circ} 32.6^{\prime}$ & $76^{\circ} 47.8^{\prime}$ & 1812.7 & 9.7 & 204 & 0.6 & 282 \\
\hline 20 & 12 & 1980 & $1250^{\mathrm{a}}$ & $26^{\circ} 25.20^{\prime}$ & $76^{\circ} 51.40^{\prime}$ & 1820.8 & 10.0 & 198 & 0.6 & 151 \\
\hline 20 & 12 & 1980 & $1446^{\mathrm{a}}$ & $26^{\circ} 6.80^{\prime}$ & $76^{\circ} 57.90^{\prime}$ & 1840.1 & 9.3 & 204 & 0.8 & 318 \\
\hline 20 & 12 & 1980 & 1545 & $25^{\circ} 58.5^{\prime}$ & $77^{\circ} 2.0^{\prime}$ & 1849.2 & 6.3 & 206 & 0.8 & 318 \\
\hline 20 & 12 & 1980 & $1548^{\mathrm{a}}$ & $25^{\circ} 58.24^{\prime}$ & $77^{\circ} 2.24^{\prime}$ & 1849.5 & 6.3 & 206 & 0.0 & 0 \\
\hline
\end{tabular}

a Satellite fix.

lard (1966), would probably result in more chaotic features.

In the abyssal depths of the Blake-Bahama Basin (Figs. 3 and 4, 0000Z, 21 October, 1980), two prominent reflectors are seen. These have been previously identified as the top of a Miocene turbidite Horizon M (6.7 s) and Horizon $\mathrm{A}^{\mathrm{u}}$ (7.1 s) (Sheridan et al., 1974; Dillon et al., 1976). No definite reflectors are seen on the Challenger profiles below Horizon $\mathrm{A}^{\mathrm{u}}$, but the two prominent reflectors, denoted $\mathrm{M}$ and $\mathrm{A}^{\mathrm{u}}$ by Dillon et al. (1976), can be traced to Site 534.

The magnetic anomaly profile (Figs. 2 and 3 ) fails to show the prominent, small-amplitude (150-gamma) Blake Spur Anomaly and the even smaller-amplitude (50-gamma) quiet-zone anomaly designated " $a$ " by Drake et al. (1963). Apparently, this is because the Challenger track crossed the Blake Spur Fracture Zone where these anomalies are broken and offset.

The same track from Site 534 to and from Fort Lauderdale, Florida, was repeated twice for the port-call interruptions on 21 October 1980 and 1 December 1980. The geophysical profiles to Fort Lauderdale pass over a small topographic expression of the buried Eleuthera Ridge at 480 n. mi. (Fig. 3). The seismic profiles reveal this as a reflection of a hummocky, mounded, seismically transparent early Miocene unit forming a partially buried ridge at $0900 \mathrm{Z}, 22$ October 1980 (Fig. 4). This unit is thought to be an extension of a serpentinelike fan complex extending northeast of the Northeast Providence Channel in the Bahamas.
The track then crosses the rough erosional topography of the mouth of Northeast and Northwest Providence Channels at 500 to $700 \mathrm{n}$. mi. (Fig. 3). Here, hard limestones, as well as chalks and the rough topography prevent penetration of seismic waves from the Challenger's small air guns. The large amplitude magnetic anomalies of the Bahama Platform are recorded (Fig. 3).

On the return track from Fort Lauderdale to Site 534, the Northern Florida Straits and the Blake Plateau are crossed (Figs. 3 and 4). The apparent ridge at $870 \mathrm{n}$. mi. (Fig. 3) is a sediment drift of Oligocene and younger sediments forming a nose off the northwest end of Little Bahama Bank. Well-stratified reflectors show about 1.0 -s thickness of sediments beneath this ridge $(0700 \mathrm{Z}$, 29 October 1980, Fig. 4). On the Blake Plateau proper only about $0.5 \mathrm{~s}$ of penetration was achieved through the transparent ooze and chalk above a harder, shallowwater limestone. Some large-amplitude magnetic anomalies (Fig. 3) over the plateau might indicate the presence of continental-type basement at depth (Taylor et al., 1968; Rabinowitz, 1974).

\section{REFERENCES}

Ballard, J. A., 1966. Structure of the lower continental rise hills of the western North Atlantic. Geophysics, 31:506-523.

Cain, J. C., Hendricks, S., Daniels, W. E., and Jensen, J. C., 1968. Computation of the main geomagnetic field from spherical harmonic expansions. Data User's Note NSSDC68-11, Tech. Rept., Goddard Space Flight Center, Greenbelt, Maryland.

Dillon, W. P., Sheridan R. E., and Fail, J. P., 1976. Structure of the western Blake-Bahama Basin as shown by 24 channel CDP profiling. Geology, 4:459-462. 
Drake, C. L., Heirtzler, J., and Hirshman, J., 1963. Magnetic anomalies off eastern North America. J. Geophys. Res., 68:5259-5275.

Heezen, B. C., and Hollister, C. D., 1971. The Face of the Deep: New York (Oxford University Press).

Rabinowitz, P. D., 1974. The boundary between oceanic and continental crust in the western North Atlantic. In Burk, C. A., and Drake, C. L. (Eds.), Geology of Continental Margins: New York (Springer Verlag), pp. 67-84
Sheridan, R. E., Golovchenko, X., and Ewing, J. I., 1974. Late Miocene turbidite horizon in the Blake-Bahama Basin. Am. Assoc. Pet. Geol. Bull., 58:1797-1805.

Talwani, M., Dorman, J., Worzel, J. L., and Bryan, G. M., 1966. Navigation at sea by satellite. J. Geophys. Res., 71:5891-5902.

Taylor, P. T., Zietz, I., and Dennis, L. S., 1968. Geologic implications of aeromagnetic data for the eastern continental margin of the United States. Geophysics, 33:755-780.

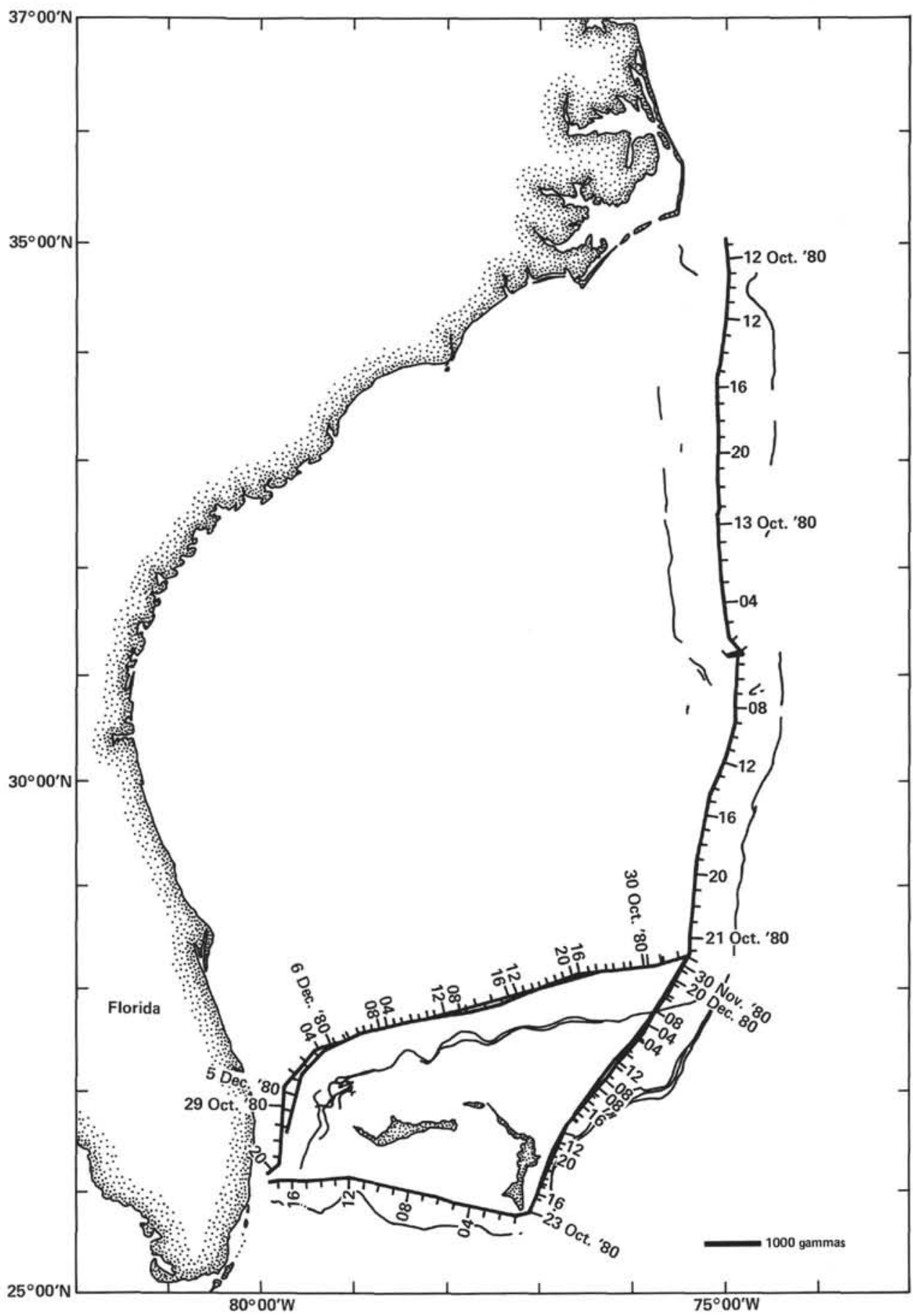

Figure 2. Track chart of Glomar Challenger during Leg 76 showing the profiles of the magnetic anomalies along the track. 

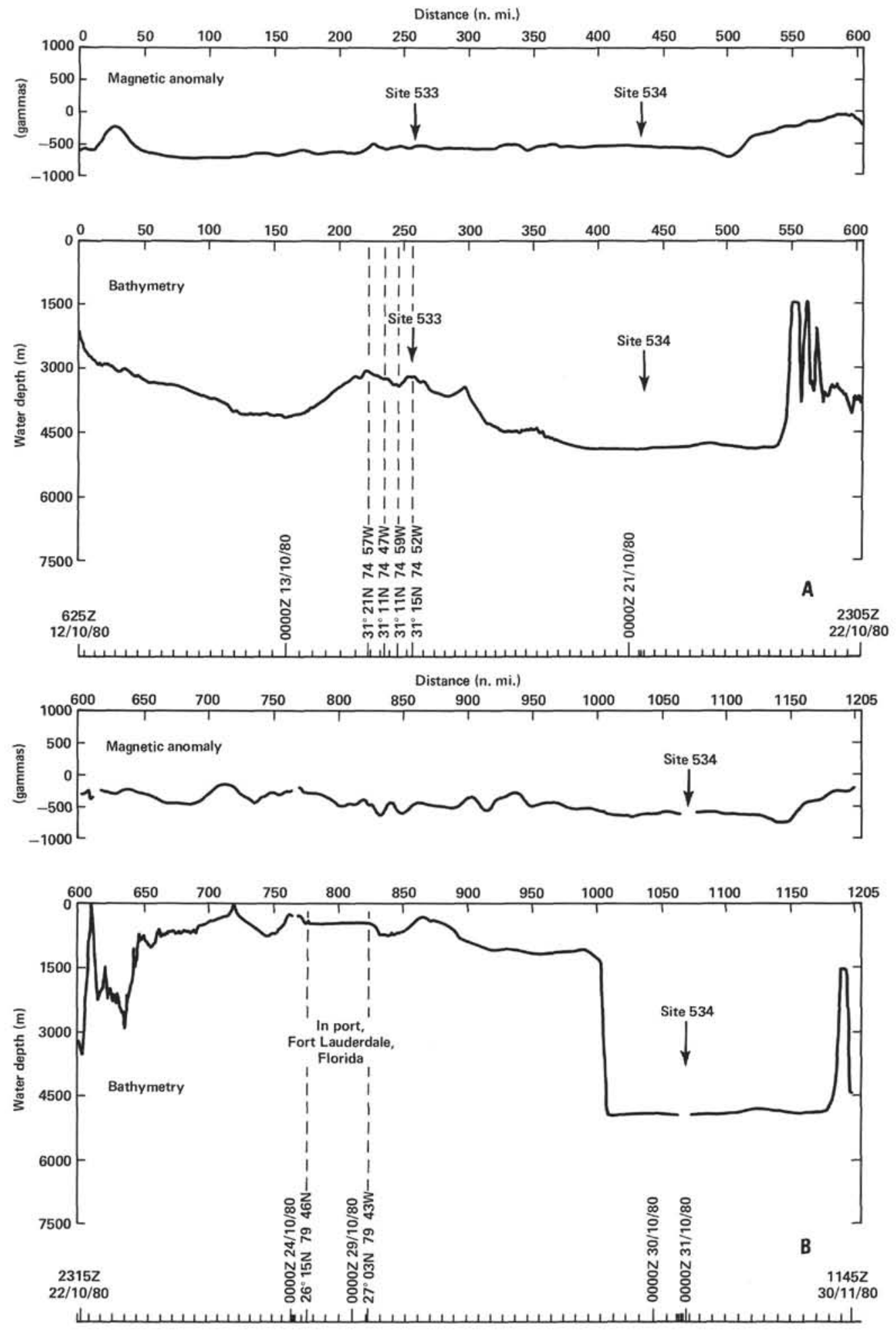

Figure 3. A-C. Magnetic anomaly and bathymetric profiles along the track of the Glomar Challenger during Leg 76. (Plots and scales are explained in the text.) 

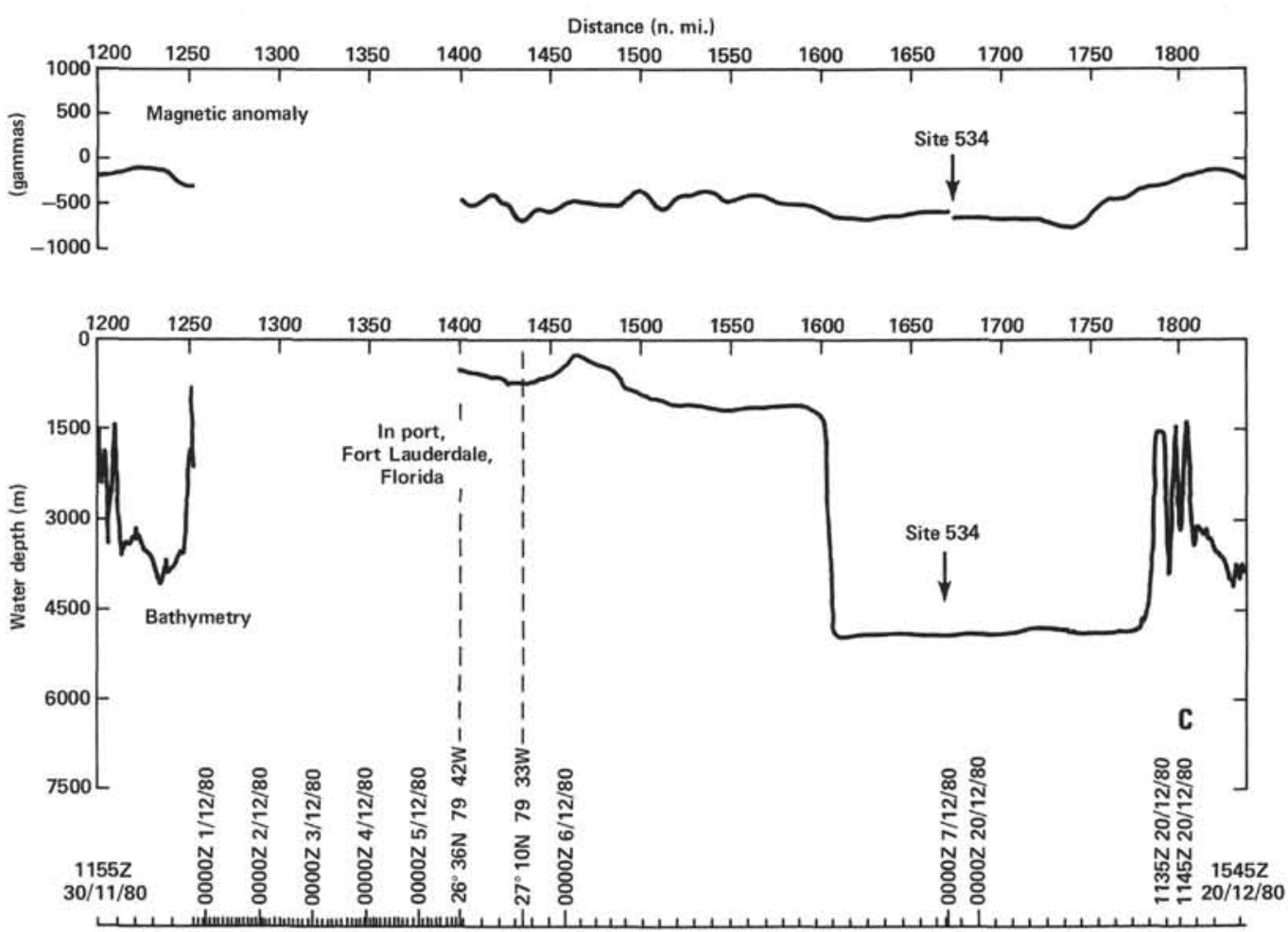

Figure 3. (Continued). 